\title{
Rôle du CD40 dans l'activation des lymphocytes B
}

L'activation et la différenciation des lymphocytes B en plasmocytes sont deux phénomènes modulés par des signaux transmis à travers la membrane cellulaire par la liaison de cytokines ou d'autres facteurs impliqués dans les interactions cellulaires. Outre les récepteurs de l'antigène, un certain nombre de molécules de surface ont été identifiées sur les cellules B. Une de ces molécules, le CD40 (membre de la famille des récepteurs du TNF) joue un rôle déterminant dans l'activation des lymphocytes B ; elle a été initialement décrite comme spécifique des cellules B [1]. A l'instar de la plupart des molécules de surface, le CD40 interagit spécifiquement avec son ligand, le L-CD40, qui est surtout exprimé à la surface des lymphocytes $T$ auxiliaires ou $\mathrm{CD}^{+}$; le L-CD40 appartient à la superfamille du TNF.

Depuis la découverte du CD40, différents groupes ont mis au point des systèmes cellulaires permettant d'étudier son intervention dans les fonctions des lymphocytes B. Ces études sont résumées dans un récent article de revue de Banchereau et al. [2]. Ainsi, la liaison d'anticorps antiCD40 aux molécules CD40 à la surface des lymphocytes $B$ induit une adhérence homotypique, la sécrétion des cytokines IL6 et IL10 et l'augmentation de l'expression de certaines molécules de surface dont celles du complexe majeur d'histocompatibilité de classe II, le CD23 et l'antigène $\mathrm{B} 7 / \mathrm{BB} 1$. Les anticorps solubles anti-CD40 sont très peu mitogènes pour les cellules $\mathrm{B}$ au repos. En revanche, ils se sont avérés très mitogènes lorsque les lymphocytes $B$ sont cultivés en présence d'activateurs polyclonaux tels que l'anti-IgM ou les esters de phorbol, effets potentialisés par l'IL4 ou l'IL13. En pré-
CD40 du ligand naturel, L-CD40, ou d'anticorps spécifiques présentés par des cellules exprimant à leur surface des récepteurs pour le fragment $\mathrm{Fc}$ d'IgG, provoque à la fois une forte prolifération et une différenciation des lymphocytes B. Ces derniers peuvent alors survivre pendant plusieurs semaines in vitro.

L'activation initiale des lymphocytes B par les lymphocytes $T$ activés dépend de contacts physiques entre les deux types cellulaires, et les cytokines produites par les lymphocytes T activés interviennent dans le maintien de cette activation dont le corollaire est la prolifération et la différenciation des lymphocytes B. L'interaction du CD40 avec son ligand naturel pourrait jouer un rôle déterminant dans ce phénomène. Tout récemment, plusieurs groupes ont confirmé cette hypothèse. Ainsi, l'inhibition de l'interaction du CD40 avec son ligand, par des anticorps spécifiques du ligand ou par la présence de CD40 soluble, inhibe l'activation des lymphocytes B par les lymphocytes $T$ auxiliaires activés [3]. De plus, les enfants atteints du syndrome hyper-IgM sont incapables de former des immunoglobulines autres que les IgM et souffrent de plusieurs affections dues à la déficience immunitaire. Chez ces malades, le gène codant pour le ligand de CD40 est muté ou présente des délétions, entraînant l'expression d'une protéine non fonctionnelle, incapable d'interagir avec le CD40 [4]. L'étude approfondie de ces malades a révélé l'absence de centres germinaux et de cellules B mémoires, suggérant ainsi un rôle capital du L-CD40 dans ce processus. Normalement, les centres germinaux se développent en follicules dans les tissus lymphoïdes secondaires et, après une stimulation antigénique, deviennent les sites de la prolifération oligoclonale des lymphocytes B ainsi que le lieu de développement des lymphocytes $B$ mémoires, spécifiques d'un antigène donné. La présence des cellules T activées est nécessaire à la formation des centres germinaux ; cependant, les mécanismes impliqués ne sont pas complètement élucidés. A cet égard, les équipes de Gray [5] et de Noelle (Dartmouth, NM, USA) [6] ont montré que le blocage in vivo de l'interaction CD40/L-CD40 après injection de la protéine de fusion sCD40- $\gamma 1$ (forme soluble du CD40) ou administration d'un anticorps anti-L-CD40 à des souris immunisées, inhibe la formation des cellules B mémoires. En revanche, certains résultats de ces deux études sont contradictoires quant au rôle dans le développement des centres germinaux de la signalisation utilisant le CD40. L'interaction du CD40 avec son ligand protège de la mort par apoptose des lymphocytes B impliqués dans les centres germinaux [7]. En revanche, la signalisation via la molécule Fas, molécule appartenant à la même famille que le CD40 et directement impliquée dans la mort cellulaire programmée, induit l'apoptose de ces cellules [8].

De nombreuses questions concernant les fonctions physiopathologiques du CD40 restent encore sans réponse. Ainsi, le CD40 est exprimé et biologiquement fonctionnel dans les cellules épithéliales du thymus [9], les monocytes et les macrophages [10] où son rôle est encore inconnu. Enfin, l'importance du CD40 dans les fonctions des lymphocytes $B$ suggère fortement que cette molécule constitue une des molécules clés dans la physiologie des lymphocytes B. 
1. Banchereau J. Interleukine 4. médecine/sciences $1990 ; 6: 946-53$.

2. Banchereau J, Bazan F, Blanchard D, et al. The CD40 antigen and its ligand. Annu Rev Immunol $1994 ; 12: 881-922$.

3. Nishioka Y, Lipsky PE. The role of CD40-CD40 ligand interaction in human $\mathrm{T}$ cell-B cell collaboration. J Immunol $1994 ; 153$ : 1027-36.

4. Di Santo J, de Saint-Basile G. Une anomalie du gène codant pour le ligand de $\mathrm{CD} 40$ est responsable du déficit immunitaire caractérisé par une hyper-IgM liée à l'X chez l'homme. médecine/ sciences $1993 ; 9$ : 456-9.
5. Gray D, Dullforce P, Jainandunsing S. Memory B cell development but not germinal center formation is impaired by in vivo blockade of CD4-CD40 ligand interaction. J Exp Med 1994 ; 180 : 141-55. 6. Foy TM, Laman JD, Ledbetter JA, Aruffo A Claassen E, Noelle RJ. gp39-CD40 interactions are essential for germinal center formation and the development of B cell memory. J Exp Med 1994 $180: 157-63$

7. Liu Y], Joshua DE, Williams GT, Smith CA, Gordon J, Maclennan ICM. Mechanisms of antigendriven selection in germinal centers. Nature 1989 342 : 929.
8. Watanabe-Fukunaga R, Brannan CI, Copeland NG, Jenkins NA, Nagata S. Lymphoproliferation disorder in mice explained by defects in Fas antigen that mediates apoptosis. Nature $1992 ; 356$ 314-7.

9. Galy AMM, Spits H. CD40 is functionally expressed on human thymic epithelial cells. J Immu nol $1992 ; 149: 775-82$.

10. Alderson MR, Armitage RJ, Tough TW Strockbine, Fanslow WC, Spriggs MK. CD40 expression by human monocytes : regulation by $c y-$ tokines and activation of monocytes by ligand for CD40. J Exp Med 1993 ; 178 : 669-74.

\section{BRÈVES}

Essai clinique d'une nouvelle classe d'hypolipidémiants, les inhibiteurs de la (HMG) CoA réductase : l'essai 4S. L'(HMG) CoA réductase catalyse la formation de mévalonate, intermédiaire engageant de façon irréversible la synthèse du cholestérol à partir de dérivés du cycle de Krebs. C'est donc l'enzyme clé de la synthèse du cholestérol contre laquelle a été développée une série d'inhibiteurs ; ils permettent d'abaisser la concentration de LDL-cholestérol dans des proportions qui n'avaient jamais été atteintes. On a pu, grâce à ces médicaments, poser enfin la question de la nocivité du cholestérol avec une chance importante de pouvoir y répondre. Un essai clinique a été réalisé dans les pays scandinaves dans le but d'évaluer l'effet d'une réduction du cholestérol sur la mortalité et la morbidité de sujets ayant une maladie cardiovasculaire (antécédents d'infarctus ou d'angine de poitrine) et une hypercholestérolémie (> 5,5 mM). Dans cet essai contrôlé, 4444 sujets ont été enrôlés, la moitié avec un traitement par simvastatin permettant le maintien de la cholestérolémie entre 3 et $5 \mathrm{mM}$, l'autre moitié recevant un placebo ; les patients des deux groupes étaient suivis au point de vue diététique. L'essai a été organisé de manière à pouvoir détecter une baisse de mortalité de $30 \%$, avec un risque de $5 \%$ et a duré en moyenne cinq ans. Une réduction de la mort d'origine cardiovasculaire de $42 \%$ a été trouvée rendant compte de la baisse de mortalité toutes causes confondues de $30 \%$. Il faut noter qu'aucune surmortalité pour une cause non vasculaire n'a été notée pour aucun des deux groupes, en particulier pas de différence dans l'occurrence de cancers digestifs ; ce qui indique que cet hypocholestérolémiant n'est pas toxique à long terme, et qu'il ne protège pas d'une autre maladie. On observe aussi une réduction de $35 \%$ des accidents cardiovasculaires non mortels chez les sujets traités qui, de plus, ont beaucoup moins recours à la revascularisation myocardique par pontage $(-37 \%, p<0,00001)$. L'effet du traitement devient apparent après un an : les lésions athéromateuses coronaires progressent moins vite, on note moins de nouvelles lésions; la diminution du risque de rupture d'une plaque athéromateuse joue sans doute un rôle important dans la baisse de la mortalité. Enfin, les femmes, bien que peu nombreuses dans cette étude, bénéficient des mêmes améliorations que les hommes.

[Scandinavian Simvastatin Survival Study group. Lancet 1994 ; 344 : 1383-9.]

Le récepteur détecteur du $\mathrm{Ca}^{2+}$ est impliqué dans certaines hypocalcémies familiales autosomiques dominantes. Le récepteur détecteur du $\mathrm{Ca}^{2+}$, couplé à une protéine $\mathrm{G}$, a été récemment identifié par $\mathrm{E}$. $\mathrm{M}$. Brown et al. (Boston, MA, USA) ; des mutations avec perte de fonction du gène codant pour ce récepteur ont été reconnues dans des familles atteintes d'hypercalcémie avec hypocalciurie et d'hyperparathyroîdie néonatale sévère $\left(m / s n^{\circ} 4\right.$, vol. $\left.10, p .475\right)$. Dans ces cas, l'hypersécrétion de l'hormone parathyroĩdienne (PTH) est due à la pente du contrôle physiologique négatif par le $\mathrm{Ca}^{2+}$. Le même groupe a étudié deux familles atteintes d'hypocalcémie autosomique dominante. Souvent l'hypocalcémie est discrète et n'entraîne pas de symptôme. Le taux d'hormone parathyroîdienne (PTH) circulante est normal (alors qu'on s'attendrait à une augmentation). En revanche, la réponse à l'administration de $\mathrm{PTH}$ exogène est normale. L'administration d'EDTA abaisse la calcémie et stimule la PTH. En utilisant comme méthode principale une digestion à la RNase, les auteurs ont mis en évidence, dans l'une des familles, une mutation faux-sens (Glu128 $\rightarrow$ Ala) dans l'exon $2 \mathrm{du}$ gène. Des études d'expression dans des ovocytes de Xenopus ont été effectuées avec l'ARN normal et l'ARN mutant. L'activation du récepteur produit une activation de la voie des inositol-phosphates. Ainsi, de plus grandes quantités d'inositol 1,4,5-triphosphate ont été détectées dans les ovocytes ayant reçu une injection d'ARN mutant que dans ceux ayant reçu l'ARN normal, exposés à une concentration basse ou élevée de calcium. Cette mutation (mutation avec gain de fonction) qui touche le domaine extracellulaire du récepteur, augmente l'activité du récepteur aux faibles concentrations de $\mathrm{Ca}^{2+}$, expliquant l'hypocalcémie chez les malades hétérozygotes. Néanmoins, une telle mutation n'a pas été retrouvée dans la seconde famille étudiée [1]. [1. Pollak MR, et al. Nature Genet 1994 ; 8 : 303-7.] 